\title{
Linc-SCRG1 accelerates progression of hepatocellular carcinoma as a ceRNA of miR26a to derepress SKP2
}

\author{
Jun-Jie Hu${ }^{\dagger}$, Cui Zhou ${ }^{\dagger}$, Xin Luo ${ }^{\dagger}$, Sheng-Zheng Luo, Zheng-Hong Li, Zi-Xin Xu and Ming-Yi Xu*
}

\begin{abstract}
Background: Increasing evidence has demonstrated that long noncoding RNAs (IncRNAs) have regulatory functions in hepatocellular carcinoma (HCC). The link between lincSCRG1 and HCC remains unclear.

Methods: To explore the lincSCRG1 regulation axis, bioinformatics, RIP and luciferase reporter assay were performed. The expressions of lincSCRG1-miR26a-SKP2 were detected in HCC tissues and cell lines through qPCR and western blot. The functions of HCC cells were investigated through in vitro assays (MTT, colony formation, transwell and flow cytometry) and the inner effect of lincSCRG1-miR26a in vivo was evaluated by xenografts and liver metatstatic nude mice models.

Results: LinCSCRG1 was found to be strongly elevated in human HCC tissues and cell lines. MiR26a and S phase kinase-related protein 2 (SKP2) were predicted as the target miRNA for lincSCRG1 and the target gene for miR26a with direct binding sites, respectively. LincSCRG1 was verified as a competing endogenous RNA (ceRNA) via negative regulation of miR26a and derepression of SKP2 in HCC cells. Both overexpression of lincSCRG1 (ovlincSCRG1) and inhibition of miR26a (in-miR26a) obviously stimulated cellular viability, colony formation, migration and proliferation of $\mathrm{S}$ phase cells and also significantly increased the protein levels of cyclinD1, CDK4, MMP2/3/9, Vimentin, and N-cadherin or inhibited the protein level of E-cadherin of HCC cells, while knockdown of lincSCRG1 (sh-lincSCRG1) and upregulation of miR26a (mi-miR26a) had the opposite effects on HCC cells. Cotransfection of inmiR26a or overexpression of SKP2 (ov-SKP2) with sh-lincSCRG1 could rescue the anticancer functions of shlincSCRG1, including suppressing proliferation and migration of HCC cells. Additionally, sh-lincSCRG1 could effectively inhibit the growth of subcutaneous xenograft tumours and lung metastasis, while the anticancer effect of shlincSCRG1 could be reversed by cotransfection of in-miR26a.
\end{abstract}

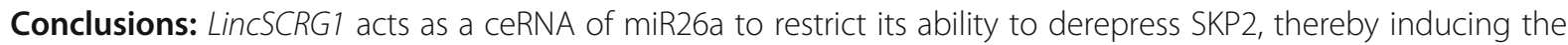
proliferation and migration of HCC cells in vitro and in vivo. Depletion of lincSCRG1 could be used as a potential therapeutic approach in HCC.

Keywords: Long noncoding RNA-SCRG1 (linc-SCRG1), microRNA-26a (miR26a), S phase kinase related protein 2 (SKP2), Hepatocellular carcinoma (HCC), Epithelial-to-mesenchymal transition (EMT)

\footnotetext{
*Correspondence: xumingyi2014@163.com

${ }^{\dagger} J u n-J i e ~ H u$, Cui Zhou and Xin Luo contributed equally to this work.

Department of Gastroenterology, Shanghai General Hospital, Shanghai Jiao Tong University School of Medicine, No 100, Haining Rd, Shanghai 200080, China
}

(c) The Author(s). 2021, corrected publication 2021. Open Access This article is licensed under a Creative Commons Attribution 4.0 International License, which permits use, sharing, adaptation, distribution and reproduction in any medium or format, as long as you give appropriate credit to the original author(s) and the source, provide a link to the Creative Commons licence, and indicate if changes were made. The images or other third party material in this article are included in the article's Creative Commons licence, unless indicated otherwise in a credit line to the material. If material is not included in the article's Creative Commons licence and your intended use is not permitted by statutory regulation or exceeds the permitted use, you will need to obtain permission directly from the copyright holder. To view a copy of this licence, visit http://creativecommons.org/ licenses/by/4.0/. The Creative Commons Public Domain Dedication waiver (http://creativecommons.org/publicdomain/zero/1. 0/) applies to the data made available in this article, unless otherwise stated in a credit line to the data. 


\section{Background}

Hepatocellular carcinoma (HCC) is regarded as the most common primary malignancy of the liver and a major cause of mortality [1]. In addition, $\mathrm{HCC}$ is not only resistant to conventional therapies but also has a high recurrence rate [2]. Thus, in view of its malignancy and the fact that its pathogenesis is still a mystery, it is necessary to elucidate the essential pathogenesis of $\mathrm{HCC}$ for the identification of novel therapeutic targets.

Long noncoding RNAs (lncRNAs) are a class of functional noncoding RNA molecules with a transcript greater than 200 nucleotides in length [3]. However, the exact biotic mechanisms and functions of most lncRNAs in HCC remain largely unknown. To date, nearly 30 deregulated lncRNAs have been identifiedas being associated with HCC [4]. Thus, lncRNAs affect cell proliferation, apoptosis and metastasis and regulatethe tumour microenvironment in $\mathrm{HCC}$, eventually causing tumour development [5].

We previously showed that lincSCRG1 was significantly upregulated in human cirrhotic livers and involved in accelerating liver fibrosis [6]. Based on this, we illustrate the role of lincSCRG1 in HCC. Our findings revealed that the lincSCRG1/miR26a/SKP2 axis is a novel regulatory mechanism implicated in $\mathrm{HCC}$ progression, which provides a potential treatment strategy for HCC.

\section{Materials and methods}

\section{Clinical specimens}

Six patients with HCC were enrolled (4 males, 2 females, age range $30-75$ years old) in the study. Six paired HCC and the corresponding adjacent liver tissues were obtained from the surgical specimen archives of Shanghai General Hospital. The diagnosis and clinical stage of $\mathrm{HCC}$ was based on clinical and histological diagnostic criteria [7]. All enrolled patients provided written informed consent and this study was approved by the ethics committee of Shanghai General Hospital.

\section{Animal experiment}

A total of $18 \mathrm{BALB} / \mathrm{c}$ nude mice (6-8 weeks old, male, $18 \pm 2 \mathrm{~g}$ ) were used (Cavens Laboratory Animal Center, Changzhou, China). All mice were randomly divided into 2 groups with SNU-387 cells $\left(2 \times 10^{6}\right.$ cells $/ 100 \mu$ l, $100 \mu \mathrm{l} /$ mouse) injected subcutaneously for the tumour xenograft model or through the tail vein for the metastasis model (each group, $n=9$ ). Then, mice in each group were randomly divided into 3 subgroups (each subgroup, $n=3$ ). Lentivirus (lentivirus titre, $10^{8} \mathrm{TU} / \mathrm{ml}, 20 \mu \mathrm{l} /$ mouse) packed with a short hairpin RNA (shRNA) of lincSCRG1(shlincSCRG1, Invitrogen, Carlsbad, CA and HanbioBiotechnology, Shanghai, China) to silence lincSCRG1, a shRNA negative control (sh-NC, Invitrogen), or sh-lincSCRG1+ miR26a inhibitor (sh-lincSCRG1 + in-miR26a, GenePharma
Co., Ltd., Shanghai, China) to silence lincSCRG1, and miR26a was injected every week for 3 continuous weeks. The sequences of the oligonucleotides used are listed in Suppl. Table 1. After 31 days, all nude mice were euthanized. The subcutaneous tumours were excised, photographed, and weighed, and the tissues were stained with immunohistochemistry (IHC) for Ki-67. The lung tissues of the metastasis model were stained with haematoxylin \& eosin (H\&E). All these procedures followed the guidelines and were approved by the Ethics Committee of Shanghai General Hospital.

\section{Cell line culture}

The human liver cell line (LO2), mouse liver cell line (AML-12) and HCC cell lines (HepG2, Hep3B, HCCL M3, SNU-387) were used. Primary hepatocytes (PHCs) were isolated from wide-type (WT) C57BL/6 mice using a two-step collagenase digestion method. Cells were cultured in Dulbecco's modified Eagle's medium (DMEM) or M199 medium (Gibco, Grand Island, NY).

\section{Lentivirus construction and transfection}

Expression vectors encoding homo sh-lincSCRG1 were used to knockdown genes,and vectors encoding homo ov-lincSCRG1 were used to overexpress lincSCRG1 (Hanbio Biotechnology). Lentivirus vectors of pLVTHM and pLV-IRES-DsRed were used to construct the expression plasmids. The virus supernatant was harvested from 293 T cells. Then, SNU-387 or Hep3B cells were transfected with lentivirus for $72 \mathrm{~h}$. The efficiency of infection was verified by fluorescence microscopy. The sequences of the oligonucleotides used are listed (Suppl. Table 1).

The full-length cDNA fragment $(1275 \mathrm{bp})$ of SKP2 (S phase kinase related protein 2) (NM_005983, Suppl. Table 1) was PCR amplified and used for genetic transformation for the overexpression experiments.

MiR26a mimic (mi-miR26a), miRNA negative control (mi-NC), miR26a inhibitor (in-miR26a) or miRNA inhibitor negative control (in-NC) was used to overexpress or knockdown miR26a (GenePharma Co., Ltd., Suppl. Table 1). SNU-387 or Hep3B cells were transfected using Lipofectamine 3000 (Invitrogen) according to the manufacturer's instructions. Then, the transfected cells were cultured $\left(37^{\circ} \mathrm{C}, 5 \% \mathrm{CO} 2\right)$ for $24 \mathrm{~h}$.

\section{Quantitative real-time PCR (qPCR)}

Total RNA was extracted from tissue and cells using TRIzol reagent (Invitrogen) according to the instructions. QPCR was performed using a SYBR Green PCR Kit (Applied Biosystems, Carlsbad, CA) and an ABI 7900HT Fast Real-Time PCR System (Applied Biosystems). The sequences of the primers used are listed in Suppl. Table 2. 


\section{Western blot analysis}

Western blotting was performed using the following antibodies: anti-cyclin D1 (1:2000), anti-CDK4/6 (cyclindependent kinases 4/6,1:2000), anti-MMP2/3/9 (matrix metalloproteinases $2 / 3 / 9,1: 1000-2000)$, anti-E-cadherin (1:500), anti-N-cadherin (1:100), anti-vimentin (1:2000), anti-SKP2 (1:500), and anti-GAPDH (1:2500), and the secondary antibody HRP-IgG $(1: 10,000)$ was used (Abcam, Cambridge, MA). The details of the antibodies are displayed in Suppl. Table 3.

\section{MTT assay}

The 3-(4,5-dimethylthiazol-2-yl)-2,5-diphenyltetrazolium bromide (MTT) assay was employed to examine the proliferation of cells. The optical density (OD) value was measured by a spectrophotometric plate reader (Thermo, Waltham, MA) at $570 \mathrm{~nm}$.

\section{Colony formation assay}

The cells were digested with $0.25 \%$ trypsin to generate a single cell suspension for counting. Cell suspensions of each group were inoculated on a dish (200 cells/dish) to continue culture $\left(5 \% \mathrm{CO} 2,37^{\circ} \mathrm{C}\right)$ until the colonies were visible. The cells were washed with PBS solution $(\mathrm{pH}$ 7.4) twice and fixed with methanol for $15 \mathrm{~min}$, and the solution was discarded. Then the samples were stained with crystal violet for $10 \mathrm{~min}$, washed with running water, and dried in air at room temperature. The number of colonies with $\geq 50$ cells was counted under the microscope.

\section{Flow cytometry}

For analysis of the cell cycle, cells were seeded in 6-well plates at $5 \times 10^{4}$ cells per well. After transfection for 48 $\mathrm{h}$, the cells were trypsinized and fixed in $70 \%$ ethanol at $-20^{\circ} \mathrm{C}$ for $24 \mathrm{~h}$. Then, the cells were stained using BD Pharmingen $^{\text {Tx }}$ PI/RNase staining (BD Pharmingen, San Diego, CA). The cell cycle distribution was analysed using an Accuri C6 flow cytometer (Becton Dickinson and Company, Franklin Lakes, NJ). The data were analysed using ModFit LT software.

\section{Transwell assay}

The cells $\left(1 \times 10^{5}\right.$ cells/well $)$ were seeded in an incubator $\left(37^{\circ} \mathrm{C}, 5 \% \mathrm{CO} 2\right)$ to cultivate for $24 \mathrm{~h}$ in a Transwell chamber (Corning Incorporation, Corning, NY) containing serum-free medium in the upper chamber and DMEM containing $10 \%$ foetal bovine serum in the lower chamber. Cells migrating to the lower chamber were fixed with $4 \%$ paraformaldehyde, stained with $0.5 \%$ crystal violet for $15 \mathrm{~min}$, washed with PBS 3 times, and photographed under a microscope.
RNA immunoprecipitation (RIP)

RIP experiments were performed according to the manufacturer's instructions (Magna RIP RNA-Binding Protein IP Kit, Millipore, Billerica, MA). Briefly, cells were lysed in $100 \mu \mathrm{l}$ of RIP lysis wash buffer. Whole cell extract $(50 \mu \mathrm{l})$ was added to mouse IgG (Abcam) and antiAgo2 (Santa Cruz Biotechnology, Inc., Santa Cruz, CA) in RIP buffer containing protein $\mathrm{A} / \mathrm{G}$ magnetic beadbound complexes. The RNA levels in the precipitates were determined by qPCR.

\section{Luciferase assay}

The wild-type or mutant fragments of homo lincSCRG1 or SKP2 3'UTR containing putative binding sites for miR26a were synthesized and cloned into the pGL3Basic reporter vector (Promega, Madison, WI). SNU-387 or Hep3B cells were harvested after transfection for 24 $\mathrm{h}$, and luciferase activity was detected by a Dual Luciferase Reporter Assay Kit (Beyotime, Shanghai, China) according to the manufacturer's instructions.

\section{Statisticalanalysis}

All experiments were repeated three times. The bar and curve graphs show the mean and standard deviation. Data were analysed using ANOVA. All statistical analyses were performed using SPSS 25.0 (SPSS, Version X; IBM, Armonk, NY). A $p$ value $<0.05$ was considered statistically significant.

\section{Results}

The expression of lincSCRG1 was substantially upregulated in both human liver tissues and HCC cell lines

To explore the role of HCC-related lncRNAs, we focused on lincSCRG1 (XLOC_004166; lnc-Hand2-2:1; located on chr4:173453013-173,520,960; transcript length $3118 \mathrm{bp}$ ). In our previous study, we found that lincSCRG1 distinctly increased along with the progression of liver fibrosis in both human liver tissues and hepatic stellate cells (HSCs) [6]. Based on the premise that most liver cancer develops from liver cirrhosis, we hypothesized that lincSCRG1 also plays a key role in HCC progression. PCR analysis indicated that lincSCRG1expression was clearly elevated in the human HCC tissues compared to the adjacent liver tissues (each group $n=6$, $p<0.05$, Fig. 1a). LincSCRG1 was significantly upregulated in HCC cell lines, including HepG2, Hep3B, HCCL LM3, and SNU-387, compared to the LO2 or AML12 cells or PHCs $(p<0.05$, Fig. 1b). In the HCC cell lines, the expression of lincSCRG1was highest in the SNU-387 cell line and lowest in the Hep3B cell line (Fig. 1b). Therefore, we concluded that lincSCRG1 is increased in human HCC liver tissues and cells. 

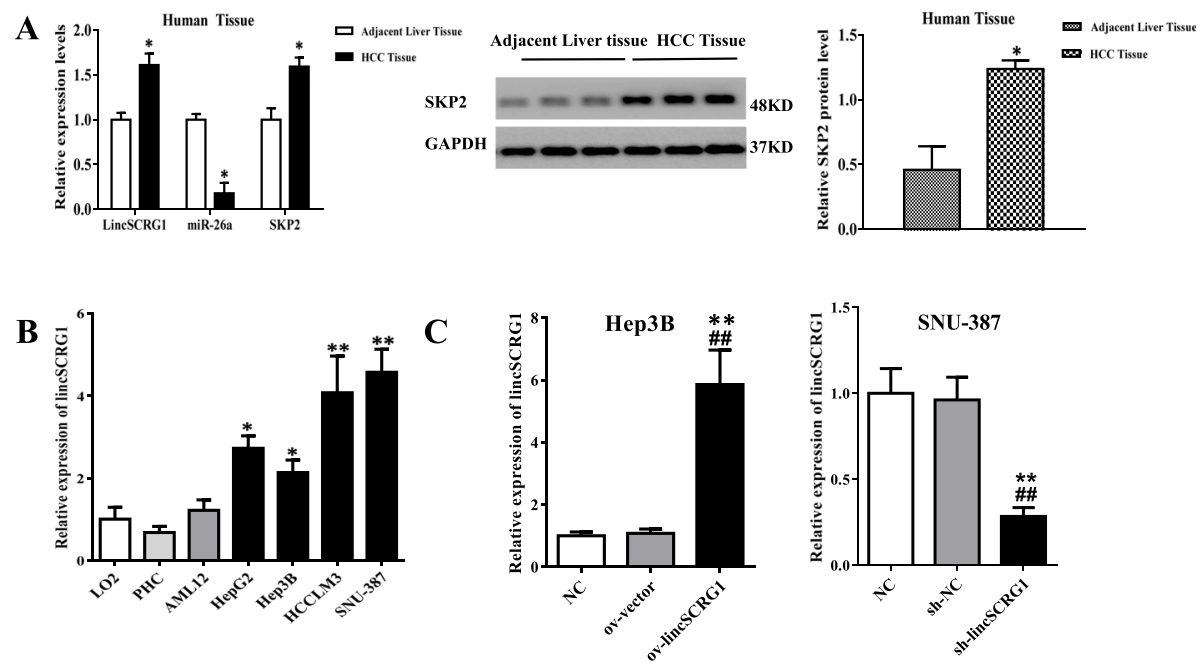

Fig. 1 Expression of lincSCRG1 was strongly upregulated in both human tissues and cell lines of HCC. a Relative lincSCRG1, miR26a and SKP2 expression was detected by qPCR (each group $n=6$ ) and the levels of SKP2 proteins were detected by western blot (each group $n=3$ ) in human HCC tissues and adjacent liver tissues. b The expression of lincSCRG1was examined in different cell lines (hepatocyte cell line [LO2, AML12], primary hepatocyte [PHC] and HCC cell lines [HepG2, Hep3B, HCCLM3, SNU-387]) by qPCR. c The overexpression efficiency of ov-lincSCRG1 in Hep3B cells and the interference efficiency of sh-lincSCRG1 in SNU-387 cells were identified by qPCR. In (a), "indicates vs. the adjacent liver tissue group, $p<0.05 ; \ln (\mathbf{b})^{* / * *}$ indicates vs. the LO2 group $\left({ }^{*}, p<0.05,{ }^{* *}, p<0.01\right)$; In (c-d $)^{* *}$ indicatesvs. The NC group, $p<0.01,{ }^{\# \#}$ indicatesvs. The ovvector/sh-NC group, $p<0.01$

\section{Overexpression of lincSCRG1 could dramatically promote the cell proliferation and migration of HCC in vitro}

Since SNU-387 cells exhibited the highest expression of lincSCRG1, while Hep3B cells displayed the lowest expression among HCC cell lines, we selected them for the following in vitro study. To evaluate the molecular function of lincSCRG1, we established sh-lincSCRG1 (in SNU-387 cells) and ov-lincSCRG1 (in Hep3B cells) cell lines. The overexpression efficiency of ov-lincSCRG1 in Hep3B cells (ov-lincSCRG1: 5.86-fold vs. NC; 5.44-fold vs. ov-vector) and interference efficiency of shlincSCRG1 in SNU-387 cells (sh-lincSCRG1: 71.6\% vs. $\mathrm{NC} ; 70.5 \%$ vs. sh-NC) were identified (Fig. 1c).

Ov-lincSCRG1 significantly increased cell viability from Day 3 to 5 in the MTT assay and promoted cell proliferation in the colony formation assay of Hep3B cells, while sh-lincSCRG1 strongly inhibited the cellular viability and proliferation of SNU-387 cells (Fig. 2a/b). A lower frequency of cells in G1 phase and a higher frequency of cells in $S$ phase were observed in the ovlincSCRG1 Hep3B cells than in the ov-NC Hep3B cells, while the opposite results were observed in the shlincSCRG1 SNU-387 cells ( $p<0.05$, Fig. 2c). However, neither ov-lincSCRG1 nor sh-lincSCRG1 influenced the percentage of cells in G2 phase (Fig. 2c). Consistently, ov-lincSCRG1 obviously increased the pro-proliferative protein cyclin D1 in Hep3B cells, while sh-lincSCRG1 inhibited the protein levels of cyclin D1 and CKD6 in SNU-387 cells (Fig. 2e). Furthermore, ov-lincSCRG1 significantly increased the migration of Hep3B cells, while
sh-lincSCRG1 had the opposite effect in SNU-387 cells (Fig. 2d). The protein levels of MMP-2/3/9 (epithelial to mesenchymal transition [EMT]-related markers) were significantly decreased in the sh-lincSCRG1 SNU-387 cells, but only MMP-2 was markedly increased in the ov-lincSCRG1 Hep3B cells (Fig. 2e). For the adhesin proteins, ov-lincSCRG1 decreased the levels of E-cadherin but elevated the levels of $\mathrm{N}$-cadherin and Vimentin in Hep3B cells, while sh-lincSCRG1 produced the opposite effects in SNU-387 cells (Fig. 2e). These results indicated that upregulation of lincSCRG1 could promote the proliferation, migration and EMT of HCC cells, while silencing of lincSCRG1 could have the opposite effects.

\section{LincSCRG1 negatively regulates miR26a by acting as a ceRNA}

LncRNAs are known to act as molecular sponges of miRNAs, also called competing endogenous RNAs (ceRNAs) for specific miRNAs, to exert their function $[8,9]$. Therefore, we hypothesized that lincSCRG1 might also interact with miRNAs as a ceRNA in HCC. The interaction probabilities between lincSCRG1 and miRNAs were assessed through the online software miRDB (http://mirdb.org/miRDB/index.html) , which showed that 50 miRNAs might be regulated by lincSCRG1 (Suppl. Table 4). Then, 7 miRNAs (miR-1297, 4465, $26 \mathrm{a} / \mathrm{b}, 4779,4778,345,203 \mathrm{~b}$ ) were identified due to low expression in tumour tissues. By searching the literature, we found that 5 miRNAs (miR26a/b, 345, 203, 1297) were reported to be involved in HCC and selected them 


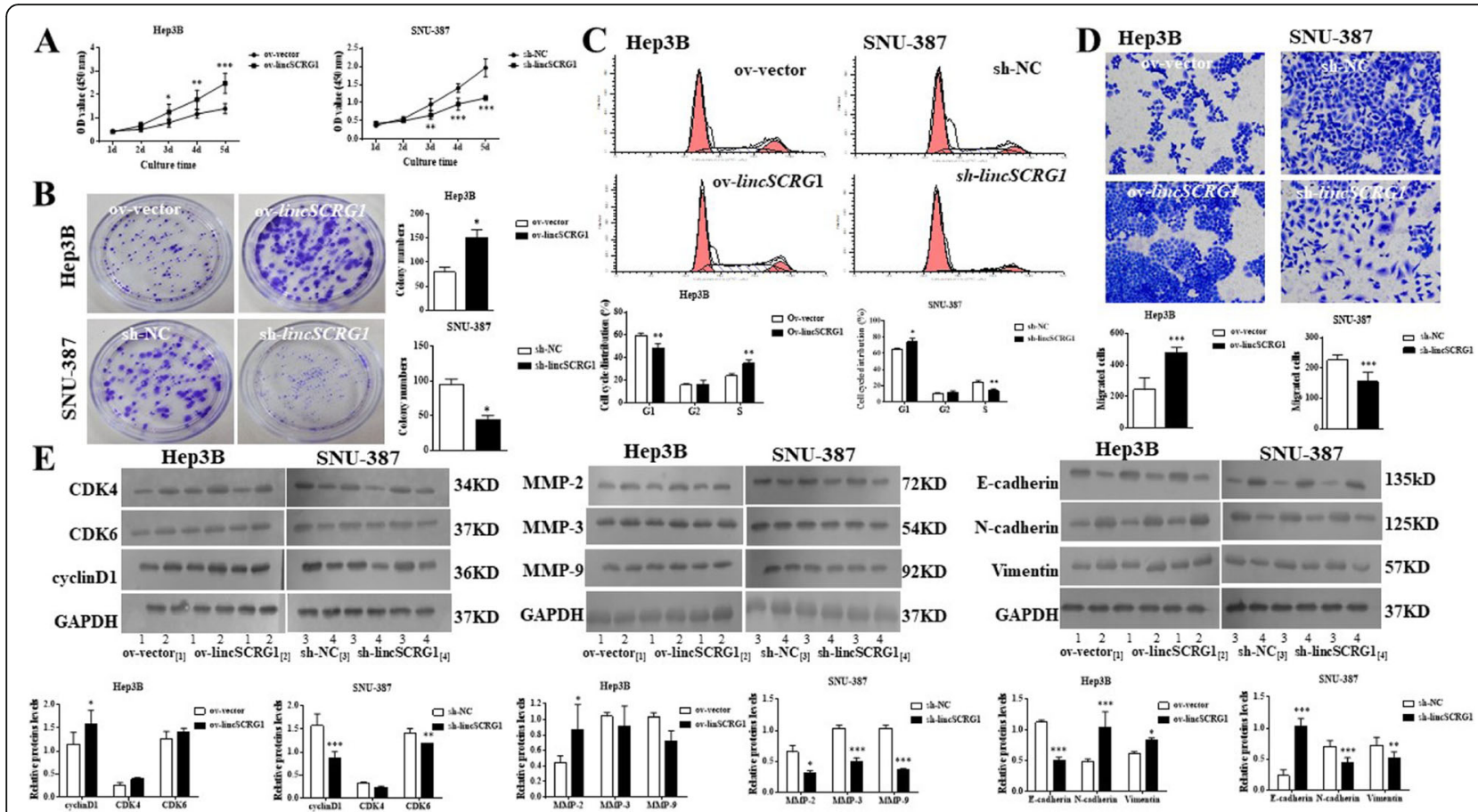

Fig. 2 Overexpression of lincSCRG1 dramatically promoted HCC cell proliferation and migration in vitro. Sh-lincSCRG1, sh-NC (in SNU-387 cells), ovlincSCRG1 and ov-vector (in Hep3B cells) cell lines were established. a Cell viabilitywas examined by MTT assays. b Oncogenic survival wasassessed by colony formation assays. c Cell cycle proliferation was evaluated by flow cytometry. $\mathbf{d}$ Migration was determined by transwell assays. e Cell cycle-related proteins (CKD4/6 and cyclinD1) and EMT-related proteins (MMP-2/3/9, E-cadherin, N-cadherin and Vimentin) were examined by western blot analysis. In (a-e), ${ }^{* / * * * * *}$ indicatesvs. The ov-vector/sh-NC group $\left({ }^{*}, p<0.05,{ }^{* *}, p<0.01,{ }^{* * *}, p<0.001\right)$

for further verification by qPCR. Only the expression of miR26a was clearly increased in the sh-lincSCRG1 SNU387 cells compared to the sh-NC cells $(2.7$-fold, $p<0.05)$ but significantly decreased in the ov-lincSCRG1 Hep3B cells compared to the ov-NC cells (0.34-fold, $p<0.05$, Fig. $3 \mathrm{~b})$. The data revealed that lincSCRG1 negatively regulated miR26a in HCC cells. In addition, Lin showed that IncRNA DLGAP1-AS1 could act as a ceRNA of miR26a to promote HCC development [10]. Taken together, these results suggest that miR26a is a target ceRNA of lincSCRG1 for further elucidation. Through bioinformatic analysis of miRDB, predicted binding sites between miR26a and lincSCRG1were identified (Fig. 3a). The expressions of miR26a were significantly downregulated in the human HCC tissues compared to the adjacent liver tissues (each group $n=6, p<0.05$, Fig. 1a). In addition, substantially decreased expression of miR26a was observed in these $4 \mathrm{HCC}$ cell lines compared to LO2 or AML12 cells or PHCs, so we selected the Hep3B and SNU-387 cell lines for further study (Fig. $3 c)$. To downregulate or upregulate miR26a, we transfected miR26a mimics, inhibitors and their individual negative controls (mi-miR26a and mi-NC, in-miR26a and in-NC) into SNU-387 and Hep3B cells (Fig. 3d). Furthermore, RIP assays showed that both lincSCRG1 and miR26a were enriched in the precipitated complex loaded with Ago2 antibodies but not IgG antibodies (Fig. 3e). The luciferase activity in the SNU-387 and Hep3B cells cotransfected with mi-miR26a and lincSCRG1-wt (wild type of lincSCRG1) was significantly reduced compared withthat in the cells transfected with mi-NC and lincSCRG1-wt, while the luciferase activity in the mimiR26a and lincSCRG1-mut (mutant type of lincSCRG1) transfection group and mi-NC and lincSCRG1-mut transfection group had no obvious difference (Fig. 3f). To further explore the competitive relationship of miR26a and lincSCRG1, we detected the relative expression of lincSCRG1 when SNU-387 and Hep3B cells were treated with miR26a mimics or inhibitors. Mi-miR26a strongly inhibited lincSCRG1 expression by approximately $70 \%$, while in-miR26a counteracted this effect by approximately 2 -fold compared to individual negative controls in both cell lines (Fig. 3g). Therefore, our data revealed that lincSCRG1 has binding sites with miR26a and negatively regulates miR26a as a ceRNA.

\section{MiR26a is negatively correlated with the proliferation and migration of HCC cells in vitro}

To explore the role of miR26a in HCC in vitro, we established 2 groups of SNU-387 cells (mi-miR26a and mi-NC group) and Hep3B cells (in-miR26a and in-NC group). Upregulation of miR26a obviously inhibited cell 


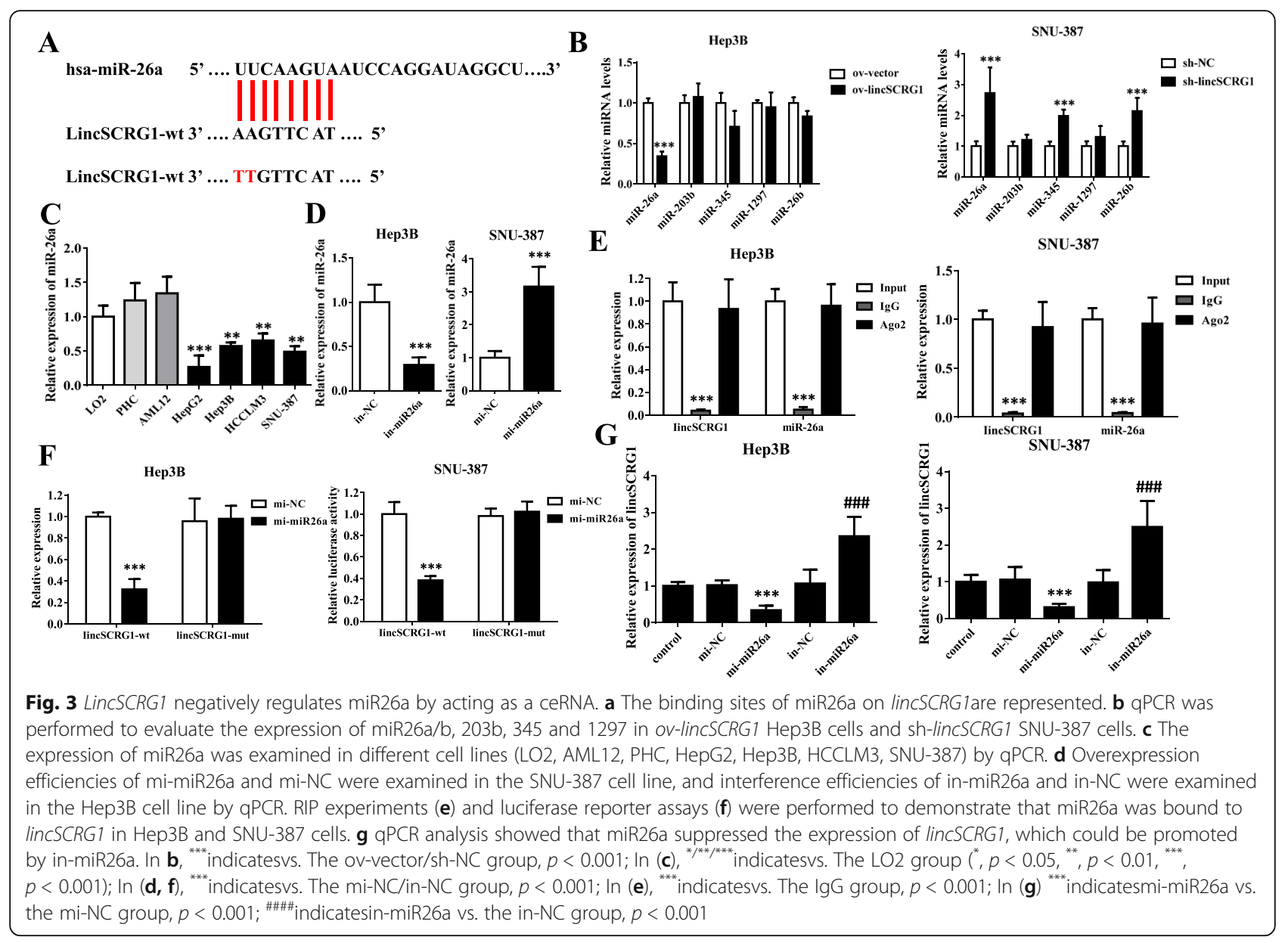

viability from Day 3 to Day 5 in the MTT assay, suppressed cell proliferation in the colony formation assay, increased the frequency of cells in G1 phase and decreased the frequency of cells in S phase in flow cytometry analysis, and decreased the migrating number of cells in the transwell assay of SNU-387 cells, while downregulation of miR26a resulted in opposite effects on Hep3B cells (Fig. 4a-d). The levels of pro-proliferative proteins (cyclin D1, CDK4) and EMT-related proteins (MMP2, Vimentin) were clearly decreased, while EMT-related proteins (E-cadherin) were evidently elevated in the mimiR26a SNU-387 cells (Fig. 4e). Opposite effects could be observed in the in-miR26a Hep3B cells (Fig. 4e). These data illustrated that overexpression of miR26a probably suppresses cell proliferation and migration of HCC cells.

\section{SKP2 is the direct target gene of miR26a, and the lincSCRG1/miR26a/SKP2 regulatory axis was identified in $\mathrm{HCC}$ in vitro}

To probe the molecular mechanisms underlying miR26a, we used miRNA target prediction programmes (TargetScan, miRDB and miRcode) and the Disgenet database to search for the candidate targets of miR26a. A total of 8 potential target genes that were identified to participate in HCC progression were selected by bioinformatics analysis. Among them, 4 target genes, SKP2, WNK1 (with-no-lysine kinase 1), MAT2A (methionine adenosyltransferase 2A) and EIF5A2 (eukaryotic translation initiation factor $5 \mathrm{~A} 2$ ), have never been reported to be regulated by miR26a. The mRNA and protein levels of SKP2 were strongly elevated in the human HCC tissues compared to the adjacent liver tissues (each group of PCR $n=6$, each group of western blot $n=3, p<0.05$, Fig. 1a). QPCR analysis verified that only the expression of SKP2 was clearly increased in the in-miR26a HCC cells compared to the in-NC cells, while it was significantly decreased in the mi-miR26a cells compared to the mi-NC cells (Fig. 5a). While the relationship between SKP2 and miR26a is still unknown, we identified SKP2 as a target gene for further study. SKP2 is the F-box protein of the E3 ubiquitin ligase complex and is responsible for targeted recognition and degradation of different cyclin-dependent kinase inhibitors [11]. Furthermore, recent studies have shown that SKP2 plays an oncogenic role in promoting tumour cell growth and 


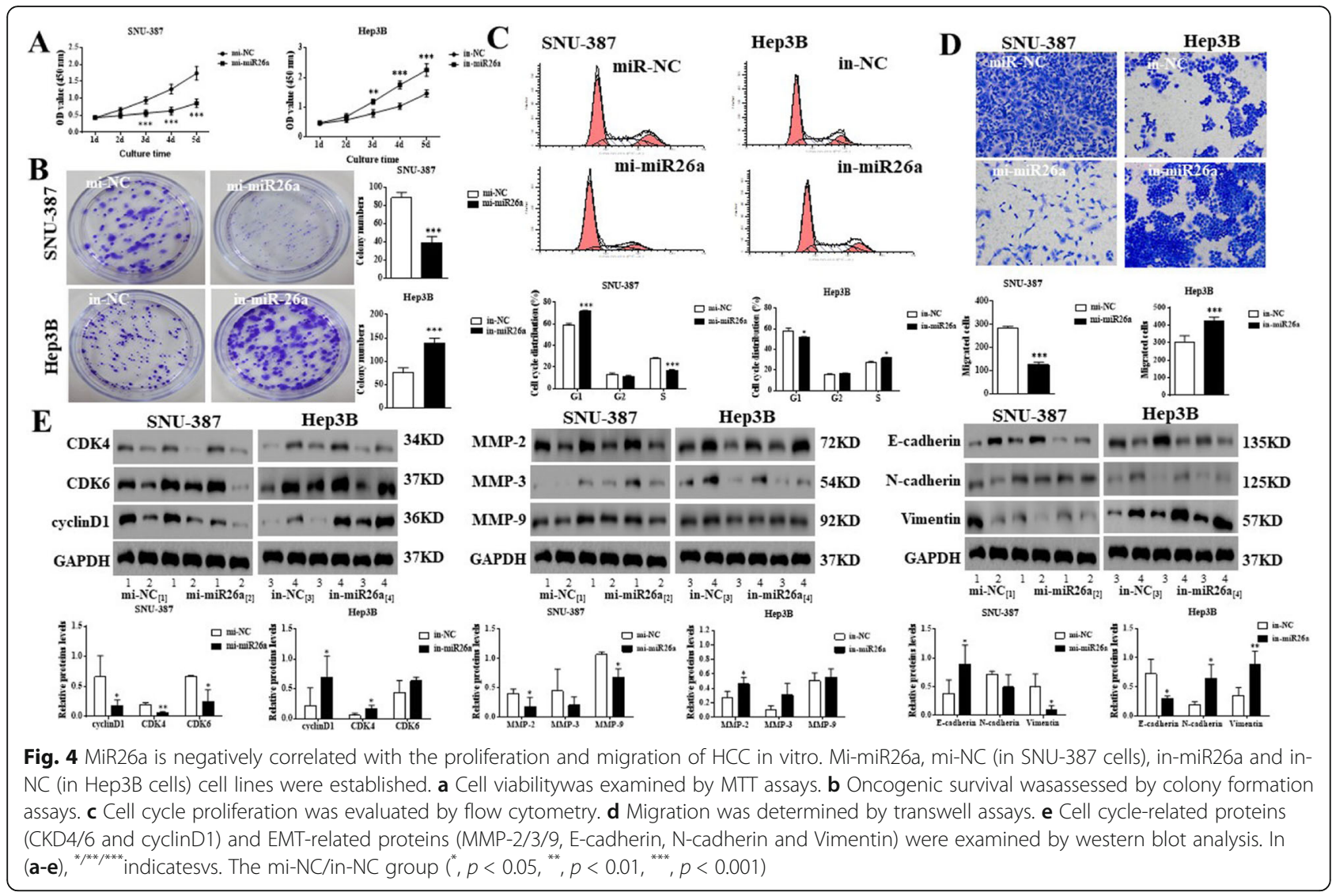

migration in HCC [12]. According to the search results, SKP2 has eight binding sites in the $3^{\prime}$-untranslated region (3'UTR) with miR26a (Fig. 5b), which indicated their higher binding affinity. To confirm that SKP2 is the downstream target of miR26a, we established a miR26a overexpression (mi-miR26a) group and a negative control (mi-NC) group in both the SNU-387 and Hep3B cells. In the mi-miR26a cells, the expression of SKP2 proteins was reduced by approximately $60-70 \%$ compared with that in the mi-NC cells in both cell lines (Fig. 5c, Fig. S1A). Consistently, mi-miR26a distinctly downregulated the SKP2 mRNA levels in both cell lines (Fig. 5c, Fig. S1A). The recognition sites or the mutant sequences of SKP2 (wild type or mutant type of SKP2 [SKP2-wt/-mut]) were subcloned into luciferase reporter plasmids in both cell lines treated with miR26a mimics or negative control. Only cotransfection with SKP2-wt and overexpression of miR26a decreased luciferase activity (Fig. 5d). Finally, to validate the regulatory function of lincSCRG1 via the miR26a/SKP2 axis, we treated SNU-387 and Hep3B cells with sh-lincSCRG1, sh-lincSCRG1 + ov-SKP2, sh-lincSCRG1 + in-miR26a and sh-NC. Sh-lincSCRG1 substantially downregulated SKP2 at both the mRNA and protein levels, while cotransfection with ov-SKP2 or in-miR26a abolished this effect (Fig. 5e, Fig. S1B). Thus, miR26a could bind to the target sites and directly downregulate SKP2. Then, we demonstrated the regulatory mechanism of the lincSCRG1/ miR26a/SKP2 axis in HCC cells.

\section{LincSCRG1 promotes cell proliferation and migration of HCC via regulating the miR26a/SKP2 axis in vitro}

Furthermore, to elucidate the effect of the lincSCRG1/ miR26a/SKP2 axis on cell growth and migration in HCC, we treated SNU-387 and Hep3B cells with shlincSCRG1, sh-lincSCRG1 + ov-SKP2, sh-lincSCRG1 + inmiR26a and sh-NC.

Interestingly, both sh-lincSCRG1 + ov-SKP2 and shlincSCRG1 + in-miR26a could effectively promote cell viability in the MTT assay (Fig. 6a), accelerate cell proliferation in the colony formation assay (Fig. 6b), decrease the frequency of cells in G1 phase and increase the frequency of cells in G2/S phase in the flow cytometry analysis (Fig. 6c), and elevate the migrating number of cells in the transwell assay (Fig. 6d) compared to sh-lincSCRG1 in SNU-387 and Hep3B cells. In addition, downregulated pro-proliferation proteins (cyclin D1, CDK4/6) and EMT-related proteins (MMPs, N-cadherin and Vimentin) and upregulated E-cadherin by sh-lincSCRG1 could also be reversed by ov-SKP2 and in-miR26a (Fig. 6e, Fig. S2). The results proved that ov-SKP2 or in-miR26a could reverse these 


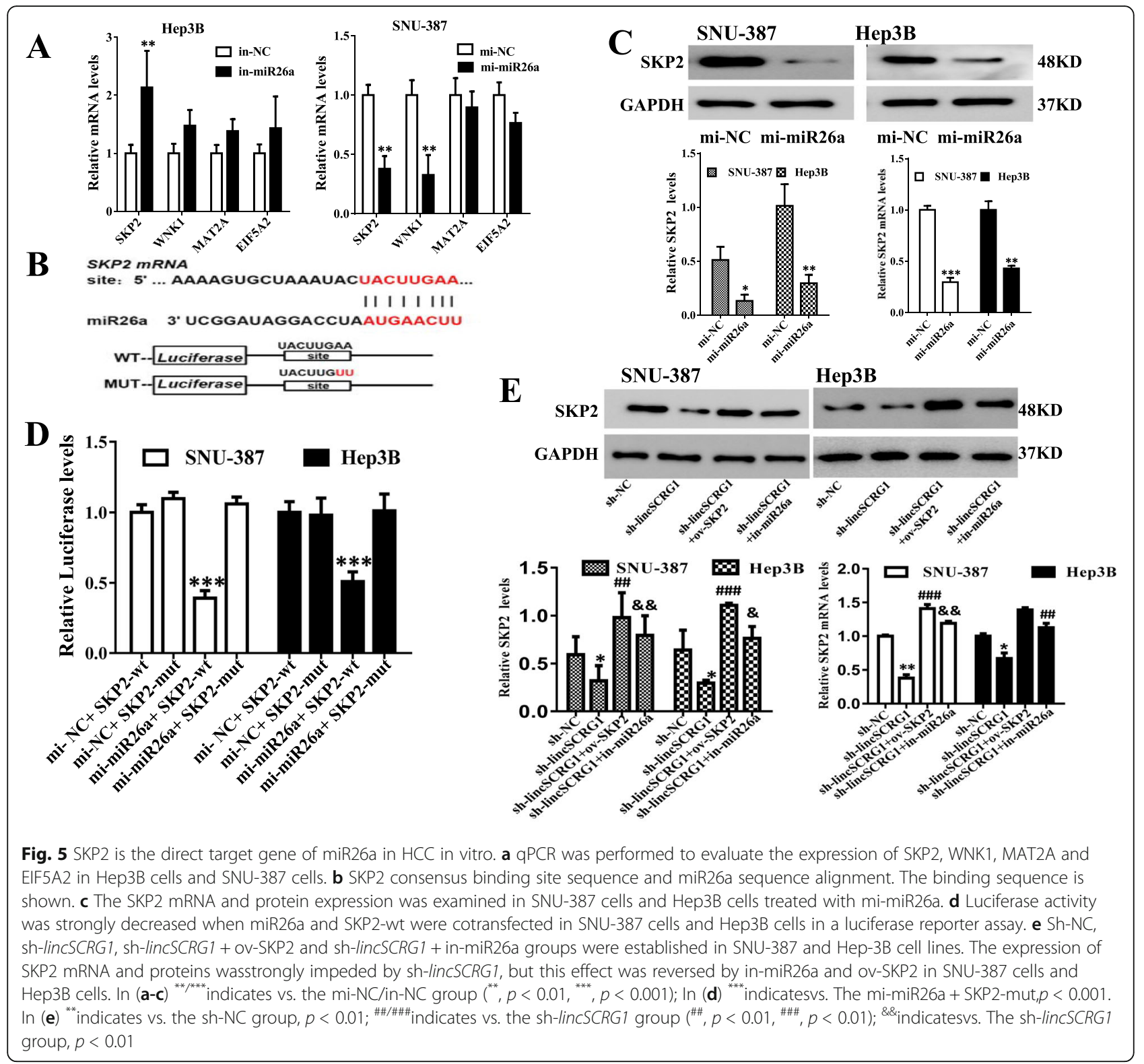

biological functions as a tumour suppressor by silencing lincSCRG1 in HCC cells, displaying the contrasting results of sh-lincSCRG1. These data suggested that lincSCRG1 promotes tumour cell growth, proliferation and migration by competitive inhibition of the target miR26a to elevate SKP2 levels, which involves the expression of proteins relevant to the cell cycle and EMT.

Silencing lincSCRG1 inhibits the growth and metastasis of $\mathrm{HCC}$, which could be reversed by miR26a

To explore the influence of lincSCRG1 on the growth and metastasis of HCC in vivo, nude mouse models were established with subcutaneousor intravenous administration of SNU-387 cells transfected with sh-NC, sh-
lincSCRG1 or sh-lincSCRG1 + in-miR26a. The tumour volume and weight of the nude mice were measured. Sh-lincSCRG1 notably inhibited tumour growth, resulting in a significantly decreased tumour volume and weightafter the 10th day in the tumour xenograft mice compared to the sh-NC mice; however, in-miR26a substantially reversed the tumour suppressor effect of shlincSCRG1 (Fig. 7a-b). In addition, IHC of Ki67 was employed to quantitively assess the proliferation index of the tissues from the xenograft tumours. Significantly fewer Ki67-positive cells were observed in the shlincSCRG1 group of tumour xenograft mice than the shNC group, while dramatically more Ki67-positive cells were observed in the sh-lincSCRG1 + in-miR26a mice than the sh-lincSCRG1 mice (Fig. 7c). Furthermore, the 


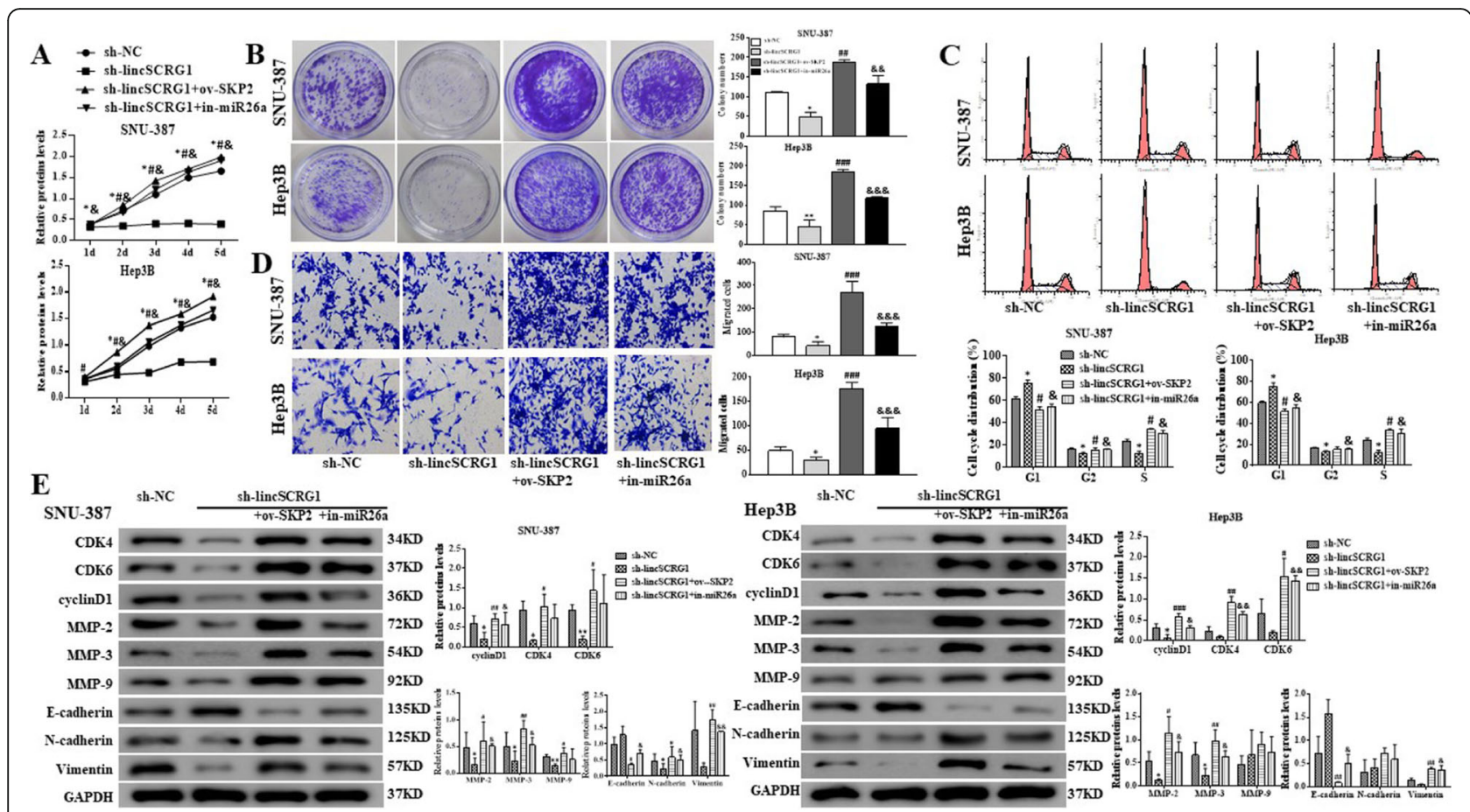

Fig. 6 LinCSCRG1 promotes cell proliferation and migration of HCC via regulating the miR26a/SKP2 axis in vitro. Sh-NC, sh-lincSCRG1, shlinCSCRG1 + ov-SKP2 and sh-lincSCRG1 + in-miR26a groups were established in SNU-387 and Hep-3B cell lines. a Cell viabilitywas examined by MTT assays. b Oncogenic survival wasassessed by colony formation assays. c Cell cycle proliferation was evaluated by flow cytometry. $\mathbf{d}$ Migration was determined by transwell assays. e Cell cycle-related proteins (CKD4/6 and cyclinD1) and EMT-related proteins (MMP-2/3/9, E-cadherin, N-cadherin and Vimentin) were examined by western blot analysis. In (a - d $)^{* / \# / /}$ indicatesthe sh-lincSCRG1 vs. sh-NC group, the sh-lincSCRG1 + ov-SKP2 vs. Sh-

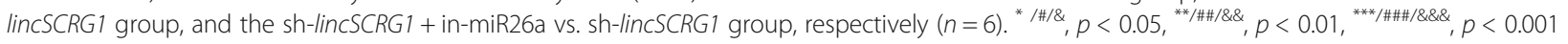

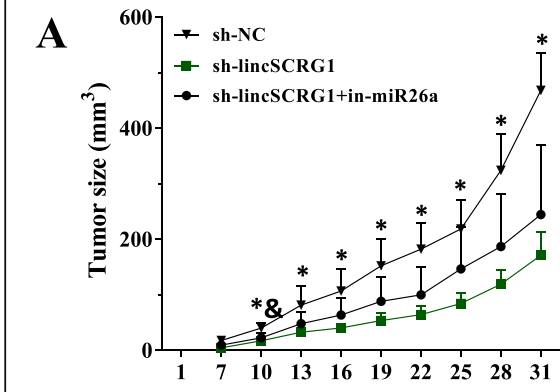

C Subcutaneous tumor tissue

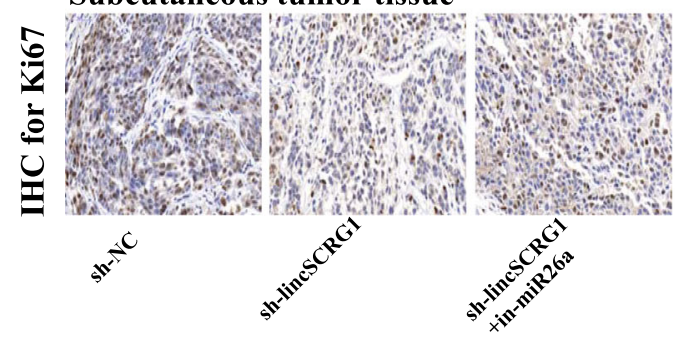

B

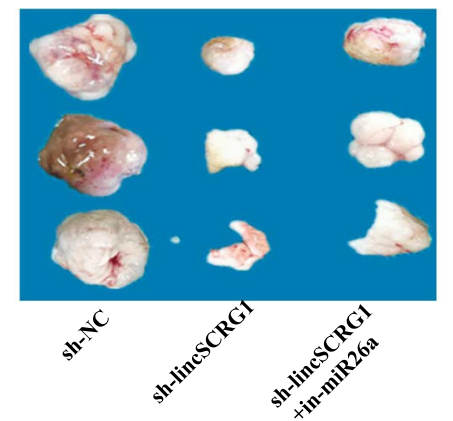

D Metastases in lung tissue

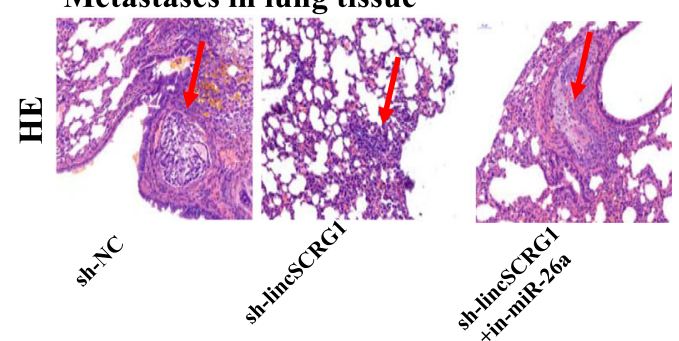

Fig. 7 Silencing LinCSCRG1 inhibitsthe growth and metastasis of HCC, which could be reversed by miR26a. Nude mouse models were established with subcutaneous or intravenous injection of SNU-387 cells transfected with sh-NC, sh-lincSCRG1 or sh-lincSCRG1 + in-miR26a. Curves of subcutaneous tumour volume (a), tumour weight (b), and IHC staining for Ki-67 in tumour tissues (c) are displayed in tumour xenograft mice (each group, $n=3$ ). $\mathbf{d}$ Lung metastasis tissue images $(400 \times)$ after HE staining in the metastasis mouse model (each group, $n=3$ ). In (a) ${ }^{*}$ indicatesthe sh-lincSCRG1 vs. sh-NC group, $p<0.05$;

\&indicatesthe sh-lincSCRG1 + in-miR26a vs. sh-lincSCRG1 group, $p<0.05 . \ln (\mathbf{b})$, ${ }^{* * *}$ indicatesthe sh-lincSCRG1 vs. sh-NC group, $p<0.001$ 
size and number of lung metastatic nodules were visibly suppressed in the sh-lincSCRG1 group of metastatic mice compared to the sh-NC group, while in-miR26a also reversed the impeded lung metastasis induced by sh-lincSCRG1 (Fig. 7d). Collectively, these data suggested that silencing lincSCRG1 could relieve tumour proliferation and metastasis in HCC, which was partially mediated by miR26a.

\section{Discussion}

According to statistics, liver cancer is one of the most common malignancies worldwide, with the eighth highest incidence rate and fourth highest mortality rate [13]. HCC is the most common histologic subtype amongmolecular subtypes (90-95\%) [14]. It is also regarded as the most common primary malignancy of the liver and a major cause of mortality [1]. However, its molecular mechanism remains a mystery; thus, identification of new molecular targets is needed to improve the therapeutic outcomes of HCC.

In recent years, a number of studies have reported that aberrant expression of lncRNAs is related to hepatocarcinogenesis [15-17]. LncRNAs, a heterogeneous group of RNA transcripts with a length of more than $200 \mathrm{nu}-$ cleotides, are generally regarded as lacking proteincoding roles [3]. The classic types of lncRNAs are scaffold lncRNAs, decoy lncRNAs, signal lncRNAs and guide lncRNAs [18]. These diverse roles determine the complex functions, including coordination of the cell state, differentiation, development and involvement of diseases [19]. Our previous study revealed thatlincSCRG1, a novel IncRNA, was upregulated along with the progression of liver fibrosis in humans by repressing the TTP-elicited inactivating effect on HSCs [6]. In this study, we found that lincSCRG1 was elevated 2.31-fold in human HCC tissues compared to the adjacent liver tissues and approximately $1.84 \sim 4.46$-fold in HCC cell lines compared to normal liver cell lines. Therefore, we hypothesized that lincSCRG1 might participate in the pathogenesis of HCC. Thus, we established ov-lincSCRG1 Hep3B cells and sh-lincSCRG1 SNU-387 cells for MTT, colony formation, flow cytometry and transwell assays to evaluate the functions of lincSCRG1 in HCC. Ov-lincSCRG1 significantly promoted cell proliferation and migration, while shlincSCRG1 presented the opposite effect. Decreases in cell cycle-related protein (cyclin D1, CDK4/6), invasionrelated protein (MMP2/3/9) and adhesion-related protein (N-cadherin, Vimentin) expression levels and increases in E-cadherin expression levels were observed in the ov-lincSCRG1 Hep3B cells, while the opposite results were observed in the sh-lincSCRG1 SNU-387 cells. Furthermore, we established an HCC model of nude mice with lincSCRG1 downregulation to assess the effect of
lincSCRG1 in vivo. We found that sh-lincSCRG1 significantly decreased the tumour size and weight of subcutaneous neoplasms and inhibited lung metastasis of HCC. These findings indicate that lincSCRG1 plays an oncogenic role in HCC by promoting cell proliferation, migration and EMT in vitro and in vivo.

LncRNAs were once considered "DNA junk" or "transcript noise", and recent studies have revealed that they could be involved in transcriptional and post-transcriptional processes and epidemic networks to perform multiplex biological functions [20]. Currently, common regulatory mechanisms of lncRNAs on miRNAs, such as functioning as miRNA precursors [21], attenuating transcription factor gene expression and transactivation [22], facilitating primary-miRNA processing [23], and acting as "miRNA sponges" or "ceRNAs" to compete with miRNA response elements to block target miRNAs, have been confirmed $[8,9]$. Among them, the latter mechanism has become a research hotspot. As lincSCRG1is an unknown noncoding RNA, we used the TargetScan algorithm to select a target ce-miRNA. We identified 7 miRNAs (miR1297, miR4465, miR26a/b, miR4779, miR4778, miR345 and miR203) that were expressed at low levels in tumour tissues or cells. Among them, miR1297/ $26 \mathrm{a} / 26 \mathrm{~b} / 345 / 203$ have been reported to be involved in HCC development. Only miR26a was significantly enhanced by sh-lincSCRG1but downregulated by ovlincSCRG1. Both luciferase reporter and RIP assays further demonstrated that lincSCRG1 had binding sites for miR26a and negatively regulated miR26a as a ceRNA. Therefore, we chose miR26a as the key target miRNA for further research. A multitude of studies have indicated that miR26a serves as a suppressor in the pathogenesis of HCC $[10,24,25]$. In our study, we also showed that inhibition of miR26a (in-miR26a) had similar effects as ov-lincSCRG1, while overexpression of miR26a (mi-miR26a) had similar effects as shlincSCRG1. In-miR26a also promoted cell proliferation and migration and decreased the protein levels of cyclin D1, CDK4/6, MMP2/3/9, N-cadherin, and Vimentin but increased the protein levels of E-cadherin in Hep3B cells, while mi-miR26a had the opposite effect in SNU387 cells. Furthermore, we verified that inmiR26a could counteract the protective effect of shlincSCRG1, including inhibition of tumour growth (tumour size or weight) in a xenograft tumour model and inhibition of lung metastasis in a mouse model. Thus, our results suggested that lincSCRG1 functions as an oncogene in HCC by competing with miR26a to inhibit the tumour suppressor effect.

Recent studies have demonstrated that miR26a could exert anticancer functions in the progression of HCC through various mechanisms, including the miR26a- 
mRNA [24], IncRNA-miR26a-mRNA [10], and cicRNAmiR26a-mRNA axis [26] and regulation of fatty acid and cholesterol homeostasis [25]. By bioinformatics analysis, we identified an innovative target gene, SKP2, with eight binding sites in the 3'UTR for miR26a. Because the relationship between SKP2 and miR26a is still unknown, our study also confirmed that SKP2 is a downstream target of miR26a. Mi-miR26a distinctly decreased the SKP2 mRNA and protein levels in HCC cell lines. Only cotransfection with SKP2-wt and mi-miR26a decreased the luciferase activity, which was not observed with cotransfection with SKP2-mut. Then, we validated the regulatory function of lincSCRG1 via the miR26a/SKP2 axis. Sh-lincSCRG1 strongly downregulated SKP2 at both the mRNA and protein levels, while cotransfection with ov-SKP2 or in-miR26a abolished this effect. Therefore, we demonstrated the regulatory mechanism of the lincSCRG1/miR26a/SKP2 axis in HCC cells. SKP2 is a cell cycle-dependent protein, and it shows low expression in the early G1 phase but is increased from G1 to S phase [27]. A recent study revealed that SKP2 not only participated in the proliferation of HCC cells through the potassium channel $\mathrm{KCa} 3.1$ but was also upregulated in poorly differentiated HCC tissue [12]. Consistent with our results, upregulation of SKP2 was also found in HCC cells, and overexpression of SKP2 (ov-SKP2) could evidently rescue the antioncogenic function of shlincSCRG1, including promoting proliferation and migration of HCC cells. Moreover, the same effects were reflected in the elevated pro-proliferation proteins (cyclin D1, CDK4/6) and EMT-related proteins (MMPs, Ncadherin and Vimentin) and reduced E-cadherin with ov-SKP2. Therefore, we speculated that lincSCRG1/ miR26a could finally promote the function of SKP2 in the cell cycle and stimulate EMT.

Interestingly, our study suggested that lincSCRG1 is a common molecular regulator for the pathological processes of fibrosis [6] and cancer genesis. Tumor microenvironment, a dynamic system orchestrated by intercellular communications, is responsible for tumor progression and metastasis [28]. Many studies from the build co-culture model have shown that HCC cells cocultured with HSCs increased tumor stemness [29], metastasis [30] and promoted HCC progression by formed the inflammatory liver cancer microenvironment. A switch from quiescent fibroblasts to cancer-associated fibroblasts (CAFs) triggers a large variety of protumorigenic signals that support tumor progression and shape the surrounding pathological stroma, with the remodelling of tissue architecture and repression of the local immune response [31]. So, we speculated that lincSCRG1 probably acts as a pro-fibrotic and protumorigenic regulator to trigger HSCs and CAFs in the pathological progression from liver fibrosis to cirrhosis, and finally carcinogenesis. The multiple functions and mechanisms are subject of further active research.

\section{Conclusions}

In summary, we showed that the lincSCRG1/miR26a/ SKP2 axis plays an important role in HCC. Briefly, lincSCRG1 could act as a ceRNA for miR26a to restrict its biological role to increase the expression of SKP2, thereby inducing the proliferation and migration of HCC in vitro and in vivo. To date, no drugs targeting lncRNAs have been applied. Our study indicated lincSCRG1 depletion as a potential therapeutic approach in HCC. Further studies need to be performed to reveal the connection between lincSCRG1 and other tumours to explore a new broad-spectrum antitumour target.

\section{Supplementary Information}

The online version contains supplementary material available at https://doi. org/10.1186/s13046-020-01825-2.

Additional file 1: Figure S1. SKP2 protein expression in HCC cells. (A) The expressions of SKP2 proteins were reduced in mi-miR26a compared to mi-NC cells in both SNU-387 and Hep3B cell lines. (B) Co-transfected of ov-SKP2 or in-miR26a with sh-lincSCRG1 could rescue the depletion of SKP2 protein inducing by sh-lincSCRG1 in 2 cell lines. Figure.S2. Different proteins expression in HCC cells. Sh-lincSCRG1 could down-regulate pro-proliferation related proteins (cyclin D1, CDK4/6) and ETM related proteins (MMPs, N-cadherin and Vimentin), and up-regulate E-cadherin, which could be reversed by co-transfection with ov-SKP2 and in-miR26a in SNU-387 and Hep3B cell lines.

Additional file 2: Suppl Table 1. Information of Plasmids used in study. Suppl Table 2. The sequence of related genes for qPCR. Suppl Table 3. The details of antibodies. Suppl Table 4. The putative microRNAs that may be regulated by linc-SCRG1.

\section{Abbreviations}

LncRNAs: Long noncoding RNAs; HCC: Hepatocellular carcinoma; SKP2: S phase kinase related protein 2; ceRNA: Competing endogenous RNA: linCSCRG1: Long noncoding RNA-SCRG1; miR-26a: microRNA-26a; ovlinCSCRG1: OverexpressinglincSCRG1; sh-linCSCRG1: Silencing lincSCRG1; ovvector: Empty vector; sh-NC: shRNA negative control; mi-miR26a: miR-26a minic; in-miR26a: miR-26a inhibitor; in-NC: miRNA inhibitor negative control; mi-miRNA: miRNA negative control; ov-SKP2: Overexpressing SKP2; shRNA: Short hairpin RNA; EMT: Epithelial-to-mesenchymal transition; IHC: Immunohistochemistry; HE: Hematoxylin-eosin; qPCR: Quantitative realtime PCR; CDK4/6: Cyclin-dependent kinases 4/6; MMP2/3/9: Matrix metalloproteinases 2/3/9; MTT: The 3-(4,5-dimethylthiazol-2-yl)-2,5diphenyltetrazolium bromide; OD: optical density; DMEM: Dulbecco's modified Eagle's medium; RIP: RNAImmunoprecipitation; HSCs: Hepatic stellate cells; lincSCRG1-wt: Wild type of lincSCRG1; lincSCRG1-mut: Mutant type of linCSCRG1; WNK1: With-no-lysine kinase 1; MAT2A: Methionine adenosyltransferase 2A; EIF5A2: Eukaryotic translation initiation factor 5 A2); 3'UTR: 3'-untranslatedregion; SKP2-wt: Wild type of SKP2; SKP2-mut: Mutant type of SKP2; CAFs: Cancer-associated fibroblasts

\section{Acknowledgements}

Not applicable.

\section{Authors' contributions}

MYX designed research and wrote the article; JJH, CZ and XL performed research and analyzed data; SZL and ZHL contributed new reagents or analytic tools; and ZXX developed software necessary to perform and record experiments. The author(s) read and approved the final manuscript. 


\section{Funding}

This study was supported by the National Natural Science Foundation of China (No. 81770597, 81570547) and the Development Program of China during the 13th Five-year Plan Period (No.2017ZX10203202003005). The funders had no role in the study design, data collection and analysis, decision to publish, or preparation of the manuscript.

\section{Availability of data and materials}

All data generated or analyzed during this study are included in this published article and its supplementary information files.

\section{Ethics approval and consent to participate}

The study protocol has been approved by the Ethics Committee of Shanghai General Hospital, Shanghai Jiao Tong University School of Medicine.

\section{Consent for publication}

Not applicable.

\section{Competing interests}

The authors hereby declare that no conflict of interest exists.

Received: 30 October 2020 Accepted: 29 December 2020 Published online: 09 January 2021

\section{References}

1. de Lope CR, Tremosini S, Forner A, Reig M, Bruix J. Management of HCC. J Hepatol. 2012;56:S75-87.

2. Liu Z, Chen D, Ning F, Du J, Wang H. EGF is highly expressed in hepatocellular carcinoma (HCC) and promotes motility of HCC cells via fibronectin. J Cell Biochem. 2018;119(5):4170-83.

3. Quinn JJ, Chang HY. Unique features of long non-coding RNA biogenesis and function. Nat Rev Genet. 2016;17(1):47-62.

4. Li C, Yang J, Liu C, Wang X, Zhang L. Long non-coding RNAs in hepatocellular carcinoma: ordering of the complicated IncRNA regulatory network and novel strategies for HCC clinical diagnosis and treatment. Pharmacol Res. 2020:158:104848.

5. Huarte M. The emerging role of IncRNAs in cancer. Nat Med. 2015;21(11): 1253-61.

6. Wu JC, Luo SZ, Liu T, Lu LG, Xu MY. Linc-SCRG1 accelerates liver fibrosis by decreasing RNA-binding protein tristetraprolin. FASEB J. 2019;33(2):2105-15.

7. Proceedings of a Bridge to a Consensus on Hepatocellular Carcinoma Management. The 2nd Asia Pacific Primary Liver Cancer Expert Meeting. July 1-3, 2011, Osaka, Japan. Oncology. 2011:81:1-164.

8. Lin Z, Zhou Z, Guo H, He Y, Pang X, Zhang X, et al. Long noncoding RNA gastric cancer-related IncRNA1 mediates gastric malignancy through miRNA-885-3p and cyclin-dependent kinase 4. Cell Death Dis. 2018;9(6):607.

9. Song $Y X$, Sun JX, Zhao JH, Yang YC, Shi JX, Wu ZH, et al. Non-coding RNAs participate in the regulatory network of CLDN4 via ceRNA mediated miRNA evasion. Nat Commun. 2017:8(1):289.

10. Lin $Y$, Jian Z, Jin H, Wei X, Zou X, Guan R, et al. Long non-coding RNA DLGA P1-AS1 facilitates tumorigenesis and epithelial-mesenchymal transition in hepatocellular carcinoma via the feedback loop of miR-26a/b-5p/IL-6/JAK2/ STAT3 and Wnt/3-catenin pathway. Cell Death Dis. 2020;11(1):34.

11. Inuzuka H, Gao D, Finley LW, Yang W, Wan L, Fukushima $\mathrm{H}$, et al. Acetylation-dependent regulation of Skp2 function. Cell. 2012;150(1):179-93.

12. Du Y, Song W, Chen J, Chen H, Xuan Z, Zhao L, et al. The potassium channel KCa3.1 promotes cell proliferation by activating SKP2 and metastasis through the EMT pathway in hepatocellular carcinoma. Int J Cancer. 2019;145(2):503-16.

13. Siegel RL, Miller KD, Jemal A. Cancer statistics, 2020. CA Cancer J Clin. 2020; 70(1):7-30.

14. Montalbano M, Rastellini C, Wang X, Corsello T, Eltorky MA, Vento R, et al. Transformation of primary human hepatocytes in hepatocellular carcinoma. Int J Oncol. 2016;48(3):1205-17.

15. He J, Zuo Q, Hu B, Jin H, Wang C, Cheng Z, et al. A novel, liver-specific long noncoding RNA LINC01093 suppresses HCC progression by interaction with IGF2BP1 to facilitate decay of GLI1 mRNA. Cancer Lett. 2019:450:98-109.

16. Ge Z, Cheng Z, Yang X, Huo X, Wang N, Wang H, et al. Long noncoding RNA SchLAH suppresses metastasis of hepatocellular carcinoma through interacting with fused in sarcoma. Cancer Sci. 2017;108(4):653-62.
17. Yi T, Wang T, Shi Y, Peng X, Tang S, Zhong $L$, et al. Long noncoding RNA $91 \mathrm{H}$ overexpression contributes to the growth and metastasis of HCC by epigenetically positively regulating IGF2 expression. Liver Int. 2020;40(2): 456-67.

18. Schmitt AM, Garcia JT, Hung T, Flynn RA, Shen Y, Qu K, et al. An inducible long noncoding RNA amplifies DNA damage signaling. Nat Genet. 2016; 48(11):1370-6.

19. Sun M, Kraus WL. From discovery to function: the expanding roles of long noncoding RNAs in physiology and disease. Endocr Rev. 2015;36(1):25-64.

20. Chen L, Zhou Y, Li H. LncRNA, miRNA and IncRNA-miRNA interaction in viral infection. Virus Res. 2018:257:25-32.

21. Augoff K, McCue B, Plow EF, Sossey-Alaoui K. miR-31 and its host gene IncRNA LOC554202 are regulated by promoter hypermethylation in triplenegative breast cancer. Mol Cancer. 2012;11:5

22. Zhai W, Sun Y, Guo C, Hu G, Wang M, Zheng J, et al. LncRNA-SARCC suppresses renal cell carcinoma (RCC) progression via altering the androgen receptor (AR)/miRNA-143-3p signals. Cell Death Differ. 2017;24(9):1502-17.

23. Chen Z, Chen X, Lei T, Gu Y, Gu J, Huang J, et al. Integrative analysis of NSCLC identifies LINC01234 as an oncogenic IncRNA that interacts with HNRNPA2B1 and regulates miR-106b biogenesis. Mol Ther. 2020;28(6):1479-93.

24. Ma Y, Deng F, Li P, Chen G, Tao Y, Wang H. The tumor suppressive miR-26a regulation of $\mathrm{FBXO11}$ inhibits proliferation, migration and invasion of hepatocellular carcinoma cells. Biomed Pharmacother. 2018;101:648-55.

25. Ali O, Darwish HA, Eldeib KM, Abdel Azim SA. miR-26a Potentially Contributes to the Regulation of Fatty Acid and Sterol Metabolism In Vitro Human HepG2 Cell Model of Nonalcoholic Fatty Liver Disease. Oxidative Med Cell Longev. 2018;2018:8515343.

26. Yang $L$, Xue $H$, Sun $Y$, Zhang L, Xue F, Ge R. CircularRNA-9119 protects hepatocellular carcinoma cells from apoptosis by intercepting miR-26a/ JAK1/STAT3 signaling. Cell Death Dis. 2020;11(7):605.

27. Wu J, Lee SW, Zhang X, Han F, Kwan SY, Yuan X, et al. Foxo3a transcription factor is a negative regulator of Skp2 and Skp2 SCF complex. Oncogene. 2013:32(1):78-85.

28. Leonardi GC, Candido S, Cervello M, Nicolosi D, Raiti F, Travali S, et al. The tumor microenvironment in hepatocellular carcinoma. Int J Oncol. 2012; 40(6):1733-47

29. Wen Q, Xu C, Zhou J, Liu NM, Cui YH, Quan MF, et al. 8-bromo-7methoxychrysin suppress stemness of SMMC-7721 cells induced by coculture of liver cancer stem-like cells with hepatic stellate cells. BMC Cancer. 2019;19(1):224

30. Coulouarn C, Corlu A, Glaise D, Guénon I, Thorgeirsson SS, Clément B. Hepatocyte-stellate cell cross-talk in the liver engenders a permissive inflammatory microenvironment that drives progression in hepatocellular carcinoma. Cancer Res. 2012:72(10):2533-42.

31. Shiraha H, Iwamuro M, Okada H. Hepatic stellate cells in liver tumor. Adv Exp Med Biol. 2020;1234:43-56.

\section{Publisher's Note}

Springer Nature remains neutral with regard to jurisdictional claims in published maps and institutional affiliations.

Ready to submit your research? Choose BMC and benefit from:

- fast, convenient online submission

- thorough peer review by experienced researchers in your field

- rapid publication on acceptance

- support for research data, including large and complex data types

- gold Open Access which fosters wider collaboration and increased citations

- maximum visibility for your research: over $100 \mathrm{M}$ website views per year

At BMC, research is always in progress.

Learn more biomedcentral.com/submission 